\title{
SYMMETRIES OF FUNDAMENTAL INTERACTIONS IN QUANTUM PHASE SPACE
}

\author{
V.V. Khruschov \\ Centre for Gravitation and Fundamental Metrology, VNIIMS, 46 Ozyornaya St., Moscow 119361, Russia
}

\begin{abstract}
Quantum operators of coordinates and momentum components of a particle in Minkowski spacetime belong to a noncommutative algebra and give rise to a quantum phase space. Under some constraints, in particular, the Lorentz invariance condition, the algebra of observables, including the Lorentz group generators, depends on additional fundamental physical constants with the dimensions of mass, length and action. Generalized symmetries in a quantum phase space and some consequences for fundamental interactions of particles are considered.
\end{abstract}

Keywords: observable; symmetry; fundamental physical constant; quantum phase space; strong interaction

PACS numbers: 11.30.Ly; 11.90.+t; 12.90.+b; 12.38.Aw

DOI: $10.1134 /$ S0202289309040069

\section{Introduction}

It is well known that the Poincaré symmetry, which is the space-time symmetry of the orthodox relativistic quantum field theory (QFT), originates from the isotropy and homogeneity of Minkowski space-time and is based on observations of macro- and microphenomena concerning conventional physical bodies and particles. For development of a general quantum theory of fundamental interactions it would be desirable to examine in detail possible generalized symmetries of fields, their interactions and the underlying space. Studies along these lines have been carried out in the context of both canonical QFT [1, 2, 3, and its modifications. Among these modifications there are theories with new fundamental physical constants other than the well known ones $c$ and $\hbar$. Beginning with Snyder's work [4, a theory with a fundamental length has been elaborated [5, 6]. However, in a theory of this sort, the reciprocity between coordinates and momenta, proposed by Born [7, was broken. This reciprocity was restored in by Yang [8, who added a fundamental mass to the modified theory.

In the present paper, we investigate the most general noncommutative algebra for quantum operators of coordinates and momentum components of a particle in Minkowski space-time under some natural conditions, in particular, the Lorentz invariance condition 9, 10. The general algebra depends on the constants with the dimensions of length [4, mass [8, and action [9]. We consider new generalized symmetries in a phase space and some consequences for funda- mental interactions of particles.

The paper is organized as follows: Section 2 is devoted to a brief description of results obtained previously for the symmetries of a quantum phase space. Some applications of these symmetries to fundamental interactions of particles are presented in Section 3. In Section 4, new generalized symmetries in a phase space and some possible consequences for the description of particle properties and their interactions are considered. Conclusions and a discussion are found in Section 5.

\section{Generalized algebra of quantum the- ory observables}

We consider a generalized algebra $g$ of quantum theory observables, when coordinates and momenta are on equal terms and form a generalized quantum phase space. The algebra $g$ generated by the observables can depend on extra fundamental constants other than the well known $c$ and $\hbar[9,10$. To restrict the considerable list of symmetries corresponding to the algebra $g$, the following natural conditions are imposed:

a) The generalized algebra $g$ of observables should be a Lie algebra;

b) The dimension of $g$ should coincide with the dimension of the algebra of canonical quantum theory observables in Minkowski space-time;

c) Physical dimensions of the observables, representing the generators of $g$, should be the same as the canonical ones; 
d) The algebra $g$ should contain the Lorentz algebra $l$ as its subalgebra and commutation relations of the generators of $l$ with other generators, should be the same as the canonical ones.

Under these conditions, the most general algebra has been derived, which depends on new constants with the dimensions of length, mass and action, and the commutation relations can be presented as $(i, j, k, l=0,1,2,3)$

$$
\begin{aligned}
& {\left[F_{i j}, F_{k l}\right]=i f\left(g_{j k} F_{i l}-g_{i k} F_{j l}+g_{i l} F_{j k}-g_{j l} F_{i k}\right),} \\
& {\left[F_{i j}, p_{k}\right]=i f\left(g_{j k} p_{i}-g_{i k} p_{j}\right),} \\
& {\left[F_{i j}, x_{k}\right]=i f\left(g_{j k} x_{i}-g_{i k} x_{j}\right),} \\
& {\left[F_{i j}, I\right]=0, \quad\left[p_{i}, p_{j}\right]=\left(i f / L^{2}\right) F_{i j},} \\
& {\left[x_{i}, x_{j}\right]=\left(i f / M^{2}\right) F_{i j},} \\
& {\left[p_{i}, x_{j}\right]=i f\left(g_{i j} I+F_{i j} / H\right),} \\
& {\left[p_{i}, I\right]=i f\left(x_{i} / L^{2}-p_{i} / H\right),} \\
& {\left[x_{i}, I\right]=i f\left(x_{i} / H-p_{i} / M^{2}\right) .}
\end{aligned}
$$

The first relation specifies the algebra $l$, while the second, third and fourth relations specify the tensor character for the well-known physical quantities. The fifth and sixth relations lead to noncommutativity of $p$ and $x$. The seventh, eighth and ninth relations generalize the Heisenberg relation. The system of relations (1) is written in units with $c=1(c$ is the velocity of light), it contains four dimensional parameters: $f$ (action), $M$ (mass), $L$ (lenght), and $H$ (action). But in the limiting case $M \rightarrow \infty, L \rightarrow \infty, H \rightarrow \infty$, the system (1) should transform to the system of relations for the canonical quantum theory, so $f=\hbar$. More generally, $f=f(M, L, H)$ and in the limiting case $f(M, L, H) \rightarrow \hbar$.

The generalized algebra (1) contains as special cases a great number of Lie algebras of different symmetry groups. The condition for the algebra (11) to be semisimple can be written in the form: $\left(M^{2} L^{2}-H^{2}\right) / M^{2} L^{2} H^{2} \neq 0$. If this condition is fulfilled, $g$ is isomorphic to a pseudoorthogonal algebra $o(p, q), p+q=6$, according to $M, L$ and $H$ values. In other cases it is isomorphic to a direct or a semidirect product of a pseudoorthogonal algebra and an Abelian or integrable algebra 9, 10. Let us display below the ranges of the $\mathrm{M}, \mathrm{L}$ and $\mathrm{H}$ parameters, which are matched to the $\mathrm{o}(3,3), \mathrm{o}(4,2)$, and $\mathrm{o}(5,1)$ algebras. If $\operatorname{sign}\left(H^{2}-M^{2} L^{2}\right)=-1, \operatorname{sign}\left(M^{2}\right)=\operatorname{sign}\left(L^{2}\right)$, or
$\operatorname{sign}\left(H^{2}-M^{2} L^{2}\right)=1, \operatorname{sign}\left(M^{2}\right)=-\operatorname{sign}\left(L^{2}\right)$, then $g=o(2,4)$. If $\operatorname{sign}\left(H^{2}-M^{2} L^{2}\right)=1, \operatorname{sign}\left(M^{2}\right)=$ $\operatorname{sign}\left(L^{2}\right)=1$, then $g=o(1,5)$, if $\operatorname{sign}\left(H^{2}-M^{2} L^{2}\right)=$ $1, \operatorname{sign}\left(M^{2}\right)=\operatorname{sign}\left(L^{2}\right)=-1$, then $g=o(3,3)$.

Irreducible representations for the pseudoorthogonal algebras of rank 3 are determined with the help of eigenvalues of three Casimir operators: $K_{1}=\varepsilon_{I J K L M N} F^{I J} F^{K L} F^{M N}, K_{2}=F_{I J} F^{I J}$, $K_{3}=\left(\varepsilon_{I J K L M N} F^{K L} F^{M N}\right)^{2}, I, J, K, L, M, N=$ $0,1,2,3,4,5$. For instance, the second-order invariant operator $K_{2}$ in terms of $I, p, x$, and $F$ can be written in the form:

$$
\begin{aligned}
& C_{2}=\sum_{i<j} F_{i j} F^{i j}\left(1 / M^{2} L^{2}-1 / H^{2}\right)+I^{2}+ \\
& \left(x_{i} p^{i}+p_{i} x^{i}\right) / H-x_{i} x^{i} / L^{2}-p_{i} p^{i} / M^{2} .
\end{aligned}
$$

The mathematical properties of the generalized algebra (1), have been studied in 9, 10, 11, 12. Apart from its mathematical properties, the algebra $g$ is an object of interest in modern physical applications as well. For instance, in [13, it is suggested to apply the algebra (11) in classical physics on astronomical scales.

\section{Some applications of the algebra $g$ to fundamental interactions of particles}

We consider possible applications of the algebra $g$ to quantum phenomena on microscales [10, 14]. In this case it is convenient to use the quantum constants $\kappa=\hbar / H, \lambda=\hbar / M, \mu=\hbar / L$ and to write the commutation relations (11) dependending on the quantum constants as (we use the natural units with $c=\hbar=1)$ :

$$
\begin{aligned}
& {\left[p_{i}, p_{j}\right]=i \mu^{2} F_{i j}, \quad\left[x_{i}, x_{j}\right]=i \lambda^{2} F_{i j},} \\
& {\left[p_{i}, x_{j}\right]=i\left(g_{i j} I+\kappa F_{i j}\right),} \\
& {\left[p_{i}, I\right]=i\left(\mu^{2} x_{i}-\kappa p_{i}\right),} \\
& {\left[x_{i}, I\right]=i\left(\kappa x_{i}-\lambda^{2} p_{i}\right) .}
\end{aligned}
$$

In the general case, one may classify Generalized Quantum Fields (GQF) as the fields which form a space for an irreducible representation of the algebra $g$ (3). For a pseudoorthogonal algebra, GQF should obey the following equation, among others:

$$
\begin{aligned}
& {\left[\sum_{i<j} F_{i j} F^{i j}\left(\lambda^{2} \mu^{2}-\kappa^{2}\right)+I^{2}+\kappa\left(x_{i} p^{i}+p_{i} x^{i}\right)-\right.} \\
& \left.\mu^{2} x_{i} x^{i}-\lambda^{2} p_{i} p^{i}\right] \Phi=0 .
\end{aligned}
$$


Let us apply the algebra (3) for a description of color particles, such as quarks or gluons. Then additional constraints arise for the form of this algebra. Due $C P$-invariance of strong interactions we obtain $\kappa=0$. Here we have used a result obtained in [9] that the commutation relations (11) became $C P$-noninvariant at finite $H$ values. Moreover, in the presence of a nonzero $\lambda$ value some inconsistencies take place in the quark descriptions inside hadrons. Thus we put $\kappa=\lambda=0$ [14. Denoting $\mu$ as $\mu_{s}$, we obtain the following nonzero commutation relations besides of the usual commutation relations with Lorentz group generators, which we do not write below:

$$
\begin{aligned}
& {\left[p_{i}, p_{j}\right]=i \mu_{s}^{2} F_{i j}, \quad\left[p_{i}, x_{j}\right]=i g_{i j} I,} \\
& {\left[p_{i}, I\right]=i \mu_{s}^{2} x_{i} .}
\end{aligned}
$$

Nonzero uncertainties immediately follow from these relations for the results of simultaneous measurement of quark momentum components. For instance, let $\psi_{1 / 2}$ be a quark state with a definite value of its spin component along the third axis. Consequently, $\left[p_{1}, p_{2}\right]=i \mu_{s}^{2} / 2$, thus $\Delta p_{1} \Delta p_{2} \geq \mu_{s}^{2} / 4$ and if $\Delta p_{1} \sim \Delta p_{2}$, one gets $\Delta p_{1}>\mu_{s} / 2, \Delta p_{2}>\mu_{s} / 2$. Rough estimations in the framework of the quark model indicate that the $\mu_{s}$ value is about $0.5 \mathrm{GeV}$. To extract a more precise number, one can use a quark equation of Dirac-Gursey-Lee type [15, 16, 14:

$$
\begin{aligned}
& {\left[\gamma_{i}\left(p_{0}^{i}+d p_{0}^{k} L_{k}^{i}+i \mu_{s} \gamma^{i} / 2\right)+\right.} \\
& \left.2 i \mu_{s} S_{i j}\left(L^{i j}+S^{i j}\right)\right] \psi=m \psi,
\end{aligned}
$$

where $p_{0}+d p_{0} L=p_{F}$ is the space-time total momentum [17] $d=\mu_{s} / m_{0}, p_{0}$ and $L$ have the forms of the usual generators of translations and Lorentz transformations in Minkowski space-time, and $p_{0}^{2}=m_{0}^{2}$. To estimate a $\mu_{s}$ value with the help of a constituent quark mass $m$ and a current quark mass $m_{0}$ values, we use a ground state $\psi_{0}$ in a meson so the contribution from $L \psi_{0}$ can be neglected. In this way we obtain from Eq.(6) the approximate relation: $m \cong m_{0}+2 i \mu_{s}$.

The constant $\mu_{s}$ should be pure imaginary and negative to account for the well-known inequality $m>$ $m_{0}$. From the correspondence between the ranges of parameters and the pseudoorthogonal groups written above, one can see that the algebra under consideration is isomorphic to the algebra of the $A d S$ group $O(2,3)$ [14.

Let us use the values of $m$ obtained in the independent quark model (IQM) on the basis of the hadron spectroscopy data [18. If we pick out from the highenergy physics data $m_{0} \cong 2 \mathrm{MeV}$ for the current $u$-quark mass and from the IQM $m \cong 316 \mathrm{MeV}$, then we obtain $\left|\mu_{s}\right| \cong 157 \mathrm{MeV}$. Thus one can evaluate the mass values of $d-, s-, c-$, and $b$-current quarks on the scale $\sim 1 \mathrm{GeV}$, which agree with their values obtained in the QCD framework [19.

\section{New generalized symmetries in phase space and their applications for description of fundamental particles}

The pseudoorthogonal groups generated with the help of the quantum operators of coordinates, momentum and angular momentum components and presented in Section 2, operate in a six-dimensional space. It should be noted that, in spite of numerous attempts, a physical meaning of six-dimensional coordinates remains unclear. Analogous problems were considered in Refs. 20, 21]. So we use the practice of embedding our $\mathrm{O}(\mathrm{p}, \mathrm{q}), \mathrm{p}+\mathrm{q}=6$, groups in more general groups that operate in a physical phase space 22,23 . In this case, two pseudoorthogonal groups $O(2,6)$ and $O(4,4)$ arise, and we argue futher in favour of their physical significance.

Actually, as has been described in Section 3 the strong interactions prefer the $O(2,3)$ symmetry which enters as a part into the $O(2,4)$ symmetry, another important physical symmetry (the conformal symmetry). The $O(2,4)$ symmetry is a part of both $O(2,6)$ and $O(4,4)$ symmetries. Moreover, if we intend to keep in mind the origin of a more general symmetry group, we should take into account the three "quantum groups" $(O(2,4), O(1,5)$ and $O(3,3))$ obtained in Section 2. The main dificulty consists in a contingency that the $O(2,4)$ group belongs to the $O(2,6)$ and $O(4,4)$ groups while the $O(1,5)$ group belongs only to the $O(2,6)$ group, and the $O(3,3)$ group belongs only to the $O(4,4)$ group.

To make a choise between these groups we take into account result obtained in [24] that indicates an association of color particles' confinement and the possible $S U(1,3)$ symmetry of nonperturbative QCD interactions. But the $S U(1,3)$ group lies only in the $O(2,6)$ group and not in the $O(4,4)$ group. Thus we suggest that, for fundamental particles, the generalized mass squared (in the natural units: $c=\hbar=1$ ) has a physical meaning:

$$
\begin{aligned}
& d f^{2}=(d E)^{2}-\left(d p_{1}\right)^{2}-\left(d p_{2}\right)^{2}-\left(d p_{3}\right)^{2}+ \\
& \mu^{4}(d t)^{2}-\mu^{4}\left(d x_{1}\right)^{2}-\mu^{4}\left(d x_{2}\right)^{2}-\mu^{4}\left(d x_{3}\right)^{2}= \\
& (d m)^{2}+\mu^{4}(d s)^{2},
\end{aligned}
$$


where the value of the constant $\mu$ is proportional to the $\mu_{s}$ value. A maximal compact subgroup of the obtained group of invariance in the physical phase space is a product of the $\mathrm{U}(1)$ and $\mathrm{SU}(4)$ groups, it is important for realizations of representations of the $\mathrm{O}(2,6)$ group or the $\mathrm{o}(2,6)$ algebra.

\section{Conclusions and discussion}

We have considered in some detail the general "quantum algebra" of the physical observables which depends on additional constants with the dimensions of mass, length and action. Generalized symmetries in a quantum phase space and some consequencies for fundamental interactions of particles have been presented. It was argued that the pseudoortogonal $\mathrm{O}(2,6)$ symmetry acting in a phase space has a physical significance.

When considering the general quantum algebra (1) we mainly restrict our treatment of generalized symmetries to the case of strongly interacting fundamental particles. This is primarily due to such an unresolved problem as confinement of color particles. Adoption of the $\mathrm{O}(2,6)$ and $\mathrm{SU}(1,3)$ groups for generalized symmetry groups of nonperturbative QCD interactions has a good potential for solving the confinement problem.

In summary, it should be said that a study of the generalized algebra (1) and the properties of solutions to Eqs. (4) and (6) are important objectives for achievement of mathematical completeness of this approach. A further investigation of the invariance group in phase space as well as possible physical applications of this invariance is in progress now and will be presented elsewhere.

\section{Acknowledgement}

The author is grateful to V.N. Melnikov, K.A. Bronnikov, V.D. Ivashchuk and S.V. Bolokhov for useful discussions.

\section{References}

[1] N.N. Bogoliubov, A.A. Logunov, A.I. Oksak, and I.T. Todorov, General principles of quantum field theory (Nauka, M., 1987 (in Russian)).

[2] D.I. Blokhintsev, Space and Time in Microcosm (Nauka, M., 1982 (in Russian)).

[3] W.I. Fushchich and A.G. Nikitin, Symmetry of quantum mechanics equations (Nauka, M., 1982 (in Russian)).
[4] H. Snyder, Phys. Rev. 71, 38 (1947).

[5] Yu.A. Golfand, JETP, 37, 504 (1959).

[6] V.G. Kadyshevsky, JETP, 41, 1885 (1961); V.G. Kadyshevsky, M.D. Mateev, V.N. Rodionov, and A.S. Sorin, Towards a maximal mass model, arXiv:0708.4205 [hep-ph] (2007).

[7] M. Born, Proc. Roy. Soc. A165, 291 (1938); A166, 552 (1938).

[8] C.N. Yang, Phys. Rev. 72, 874 (1947).

[9] A.N. Leznov and V.V. Khruschov, A general form of commutation relations of discrete space-time theory, prep. IHEP 73-38, Serpukhov (1973).

[10] V.V. Khruschov, Measurement Techniques, 35, N11, 1247 (1992), 37, N7, 717 (1994); V.V. Khruschov, in Proc. of the XV Workshop "Problems on High Energy Physics and Field Theory" (Protvino, July 1992), p.114 (IHEP, Protvino, 1995); V.V. Khruschov and A.N. Leznov, Grav. Cosmol. 9, 159 (2003) arXiv:hep-th/0207082.

[11] R. Vilela Mendes, J. Phys. A27, 8091 (1994).

[12] C. Chryssomalakos and E. Okon, Int. J. Mod. Phys. D13, 2003 (2004) arXiv:hep-th/0410212.

[13] A.N. Leznov, What our world might be like (as alternative to Minkowski space-time with Poincare group of motion), arXiv:0803.4289 [physics.class-ph] (2008).

[14] V.V. Khruschov, Grav. Cosmol. 13, 259 (2007); Generalized kinematical symmetries of quantum phase space, talk at the XIII Intern. Conf. "Selected Problems of Modern Theoretical Physics", arXiv:0807.2532 [hep-th] (2008).

[15] P.A.M. Dirac, Ann. Math. 36, 657 (1935).

[16] F. Gursey and T.D. Lee, Proc. Nat. Sc. Sci. 49, 179 (1963).

[17] C. Fronsdal, Rev. Mod. Phys. 37, 221 (1965).

[18] V.V. Khruschov, V.I. Savrin, and S.V. Semenov, Phys. Lett. B525, 283 (2002); V.V. Khruschov, Calculations of meson mass spectra in the model of quasi-independent quarks, talk at the XLI PNPI Winter School, Repino, hep-ph/0702259(2007). 
[19] C. Amsler et al. (PDG), Phys.Lett. B667, 1 (2008).

[20] M. Toler, Phys. Rev. D70, 024006 (2004) arXiv:hep-th/0305121.

[21] A.N. Leznov, Kinematic in $S U(2,2) \simeq O(2,4)$ alternative world (observable coordinates and impulses representation) arXiv:0809.1641 hepth] (2008).

[22] W.P. Shchleich, Quantum optics in phase space (Wiley-VCH Verlag, Berlin, 2001).

[23] C.K. Zachos, D.B. Fairlie, and T.L. Curtright (Eds.), Quantum mechanics in phase space (WS, Singapore, 2005).

[24] V.V. Khruschov, Confinement and U(1,3) symmetry of color particles in complex phase space, arXiv:hep-ph/0311346v2 (2004). 